\title{
L'impact De La Création Des PME/PMI Sur L'idh En Algérie « Étude Économétrique 2001-2013 »
}

\author{
Dr. Bouchikhi Mohamed Rédha \\ Maitre de Conférences, HDR en Economie \\ Faculté des Sciences Economiques, \\ Commerciales et des Sciences de Gestions Université de Mascara Algérie \\ Laboratoire : PME recherche et innovation \\ Dr. Yagoub Mohamed \\ Maitre de Conférences, HDR en Economie \\ Faculté des Sciences Economiques, \\ Commerciales et des Sciences de Gestions Université de Mascara Algérie \\ doi: 10.19044/esj.2017.v13n25p251 URL:http://dx.doi.org/10.19044/esj.2017.v13n25p251
}

\begin{abstract}
One of the major problems faced by the Algerian economy is sustainability. This is because the economy is essentially hydrocarbon-based. Since 2001, Algeria has adopted a new strategy to strengthen and diversify the economic sector in order to reduce poverty and ensure sustainable development.

This study aims to highlight the impact of these reforms on the development and the level of well-being. For this purpose, we carried out an econometric study over the period (2001-2013) in order to ascertain the impact of the creation of SMEs on the improvement of the quality of life which is measured in this test by the HDI. We found that the strategy adopted by the Algerian state for the creation and promotion of SMEs in Algeria has a positive impact on the state of development of the country and the level of well-being of the population.
\end{abstract}

Keywords: Human development, poverty, SMEs HDI

\section{Résumé}

Le problème que rencontre l'économie Algérienne, est la durabilité, étant donné que cette économie repose essentiellement sur les hydrocarbures, depuis 2001, l'Algérie a adopté une nouvelle stratégie pour renforcer et diversifier le secteur économique, afin de réduire la pauvreté et assurer un développement durable. 
Cette étude vise à mettre en lumière l'impact de ces reformes sur le développement et le niveau de bien-être. Pour cela nous avons réalisé une étude économétrique sur la période (2001 -2013), afin de voir l'impact de la création des PME/PMI sur l'amélioration de la qualité de vie qui est mesuré dans cet essai par l'IDH. Nous avons trouvé que la stratégie adoptée par l'état Algérien pour la création et la promotion des PME/PMI en Algérie à un impact positif sur l'état du développement du pays et le niveau de bien être de la population.

Mot-clés: Développement humain, Pauvreté, PME/PMI, IDH

\section{Introduction}

Tous les pays du monde vise à garantir le bien être en améliorant leurs niveau de vie, sauf que Le bien-être n'est pas chiffrable avec précision, mais il existe des indicateurs numériques qui permettent de mesurer les différentes composantes qui reflètent le bien-être. (Romina Boarini, Åsa Johansson \& Marco Mira d'Ercole, 2006)

Depuis 2001 L'état Algérien a mis en œuvre des stratégies pour améliorer le bien-être, et cela en assurant la scolarisation de tous les enfants, l'éradication de l'analphabétisme, l'amélioration de la santé, ce qui est conforme aux objectifs de l'ONU mis en place en Septembre 2001(Philippe Deubel, 2008), et l'une des stratégies employées dans ce contexte était le développement du secteur hors hydrocarbures (Djemai Sabrina, 2013).

Il n'est donc pas surprenant que les stratèges politiques ont souvent pensé que les PME/PMI peuvent devenir la «semence» de relance économique.

A partir de là, la lutte contre la pauvreté pour l'Algérie est soutenu par les politiques de développement (Khaled Sadaoui \& Mokhtar Maazouz, 2009) et comme les PME/PMI sont considérées comme moteur de croissance économique, (Ludovica Ioana Savlovshi \& Nicoleta Raluca ROBU, 2011), l'état Algérien a mis en œuvre une série de réformes pour la création et la promotion des PME/PMI.

Notre principale problématique est la suivante:

Quel est l'impact des PME/PMI sur l'amélioration du niveau de bien être en Algérie, et est-ce que les réformes portées à leur égard ont donné les résultats espérés?

\section{Etat des lieux sur les PME/PMI en Algérie}

La micro-finance est devenue un outil primordial dans la lutte contre la pauvreté mais aussi un outil dans les politiques de développement durable (Betty Wampfler, 2004). 


\section{Les contraintes de l'évolution des PME/PMI en Algérie}

En Algérie la promotion des PME/PMI a été élaboré par la loi 18-01 du 12/12//2001 portant sur 1'orientation et la promotion, sauf qu'aujourd'hui les PME/PMI Algérienne sont confrontées à des difficultés qu'on pourrait résumer dans les points suivants :

- $\quad$ Contraintes administratives

Le climat administratif Algérien est défavorable pour la croissance des PME/PMI, car le circuit est souvent jugé comme étant lent et complexe, malgré les premiers effets de la stratégie de libéralisation précoce dans le cadre de l'ajustement structurel, l'économie algérienne reste très contrôlée.

\section{- $\quad$ Contraintes de financement}

En Algérie une ambiance de manque de confiance règne entre les banques et les PME/PMI, car les banques trouvent qu'elles prennent trop de risques en finançant les PME/PMI du fait de la faiblesse de leurs capitaux d'une part, d'autre part la qualité de l'information fournie par ces PME/PMI est médiocre, soit en ex-ante ou en ex-post d'où souvent des situations d'asymétrie informationnelle assez préjudiciables pour les banques.

En contrepartie les PME/PMI trouvent que les banques représentent un obstacle à leurs croissances car elles prennent trop de temps pour l'étude de leur demande de financement et exigent beaucoup de garanties.

\section{- $\quad$ Contraintes foncières}

Il est difficile de trouver une assiette pour construire une entreprise qui pourra par le temps être agrandi, les jeunes entrepreneurs se trouvent souvent devant l'obligation de louer ou de transformer une partie de leurs maisons en atelier.

- $\quad$ Contraintes fiscales et douanières

Les impôts sont souvent jugés comme trop élevés par les entrepreneurs, ainsi que les taxes douanières qui sont souvent liées à l'importation. Aussi les lenteurs administratives en matière de dédouanement, cela influencent négativement la productivité (Farida Merzouk, 2010).

\section{- $\quad$ Contraintes d'informations}

On réalise qu'on Algérie on a un manque de données liées aux études de marché, aux opportunités d'investissement, la concurrence et les pratiques des opérateurs...etc.

En général, les PME/PMI Algériennes sont aujourd'hui prédominées par le secteur informel, leurs activités sont souvent caractérisées par la non déclaration ou la sous déclaration des ressources humaines et financières, la fraude fiscale, non facturation, surfacturation ou sous facturation ....etc.(Karim Si Lekhal, Youcef Korichi, \& Ali Gaboussa, 2013). Les contraintes et les difficultés que les PME/PMI affrontent au quotidien les poussent à exercer clandestinement. 


\section{Évolution des PME/PMI en Algérie et leurs impacts}

- $\quad$ l'évolution des PME/PMI dans les différents secteurs d'activité

Les activités des PME/PMI s'élargissent et occupent plusieurs secteurs comme nous le montre le tableau ci-dessous:

Tableau $\mathrm{N}^{\circ} 1$. L'évolution des PME/PMI dans les différents secteurs d'activité

\begin{tabular}{|c|c|c|c|c|c|}
\hline Secteur d'activité & $\begin{array}{c}1^{\text {er }} \text { semestre } \\
2012\end{array}$ & Part (\%) & $\begin{array}{c}1^{\text {er }} \text { semestre } \\
2013\end{array}$ & Part (\%) & $\begin{array}{c}\text { Evolution } \\
(\%)\end{array}$ \\
\hline Agriculture & 4142 & 1.02 & 4458 & 1.01 & 7.63 \\
\hline BTPH & 139875 & 34.3 & 147005 & 33.26 & 5.1 \\
\hline $\begin{array}{c}\text { Industrie } \\
\text { manufacturières }\end{array}$ & 65859 & 16.5 & 70840 & 16.03 & 7.56 \\
\hline Service & 195889 & 48.04 & 217444 & 49.2 & 11 \\
\hline
\end{tabular}

Source : bulletin d'information statistique de la PME/PMI - ${ }^{\text {er }}$ SEMESTRE 2013.

Comme nous le remarquons, le secteur des services détient la plus grande part avec 195859 PME/PMI en 2012 et 217444 en 2013 soit une évolution de $11 \%$, généralement ce sont des entreprises de transport ou des cybercafé.etc. ensuite vient le secteurs des bâtiments travaux publiques et hydropiques avec 139875 PME/PMI en 2012 et 147005 EN 2013 avec une évolution de $5.1 \%$ aussi cela revient au fait que l'état a besoin de ce genre d'entreprise étant donné qu'il a besoin de constructions d'infrastructures et de structures de base, l'industrie manufacturière vient ensuite avec une évolution de $7.56 \%$ dans le nombre d'entreprises qui a évolué de 65859 à 70740 PME/PMI enregistré de 2012 à 2013, mais nous remarquons que le secteur de l'agriculture détient la plus faible part de l'ensemble des secteurs avec $1.02 \%$ en 2012 et $1.01 \%$ en 2013 , ce secteur est délaissé par les PME/PMI malgré que l'Algérie a des ressources naturelles qui lui permettent de se développé dans l'agroalimentaire.

- $\quad$ L'impact des PME/PMI sur l'emploi

L'évolution des emplois est en parallèle avec l'évolution des projets financés, tant que le dispositif ANSEJ (Agence Nationale de soutien à l'emploi de Jeunes) finance la création de nouvelles entreprises, des emplois sont créés simultanément comme nous le tableau au-dessous.

Tableau $\mathrm{N}^{\circ}$ 2. L'évolution des projets financés par l'ANSEJ et des emplois créés

\begin{tabular}{|c|c|c|}
\hline Année & Projets financés & Emplois \\
\hline Au 31/12/2007 & 86380 & 243308 \\
\hline 2008 & 10634 & 31418 \\
\hline 2009 & 20848 & 57812 \\
\hline 2010 & 22641 & 60132 \\
\hline 1011 & 42832 & 92682 \\
\hline 2012 & 65812 & 129203 \\
\hline 2013 & 43039 & 96233 \\
\hline Total & 292186 & 710788 \\
\hline
\end{tabular}

Source : tableau réalisé à partir de la base des données ANSEJ. 
D'après le tableau ci-dessus, on remarque que le nombre d'emplois généré par le dispositif ANSEJ à augmenter, cela jusqu'à l'année 2012 sauf qu'en 2013 le nombre d'emploi a chuté ce qui peut être traduit par le fait que la sélection des projets est devenue plus stricte et de plus en plus de projets ont été refusés par les banques.

\section{Etat des lieux sur le bien-être et le développement en Algérie la lutte contre la pauvreté en Algérie}

Depuis la crise pétrolière en 1986, les premiers signes de la pauvreté sont apparus en Algérie cela était dû à l'effondrement des courts des hydrocarbures (Ahmed Smahi, Samir Maliki, \& Salah Eddine Arifi, 2011), le nombres des Algériens vivant avec moins de $1 \$$ par jour dépassait les 70000 personnes, la plus part vivait dans les zones rurales (Khaled Sadaoui \& Mokhtar Maazouz, 2009), sauf qu'après les années 2000 les indicateurs sociaux se sont nettement améliorés, l'espérance de vie à la naissance est passé de 71.7 ans à 75 ans de 1998 à 2010, le taux de scolarisation des enfants entre 5 et 15 ans s'est amélioré de 1995 à 2010 de $87.5 \%$ à $95 \%$, le programme de la relance économique à aussi contribuer à l'amélioration du niveau de bien être et cela en réduisant le chômage et par l'évolution de la consommation des ménage de 4\% par an en moyenne, et même l'objectif qui était censé être atteint a vu le jour en 2003 qui consister à réduire la population sous alimentés c'est-à dire attendre le but d'assurer 2100 calorie par personne par jour.

Et parmi les dispositifs mit en place par l'état Algérien pour la lutte contre la pauvreté ; on note essentiellement:

- $\quad$ Dispositif du filet social : et cela en donnant des aides financières au plus démunies.

- $\quad$ Le programme de lutte contre le chômage : par l'aide à l'insertion des chômeurs à la vie active et par des dispositifs d'incitation à la création de l'auto emploi et cela par le dispositif micro entreprises de l'ANSEJ, dispositif micro crédit de l'ANGEM, dispositif de soutien aux chômeurs promoteurs de la CNAC.

- $\quad$ Les actions de solidarité nationale, comme par exemple le soutien des enfants scolarisés issus de familles démunies, et la création du fond de la Zakat en 2003.

- Le programme de développement communautaire, ce programme consiste à aider à améliorer les conditions de vie des sites les plus démunies et cela en les aidant à construire des infrastructures de base et ceci est géré par l'Agence de Développement Social.

- $\quad$ Assistance aux populations vivant en sites précaires : cela par des compagnes de sensibilisation et des actions de santé préventives (PNUD, 2000). 


\section{aperçu sur le développement en Algérie}

Après que le système du protectionnisme à échouer, la nouvelle stratégie adopté par l'état Algérien été l'ouverture commerciale, sauf que pour ce pays le seul secteur exportateur était le secteur des hydrocarbures (Mehdi Abbas, 2012) et quand en parle de développement, on parle de durabilité (Philippe Deubel, 2008), donc il fallait penser à quelque chose pour penser aux générations avenir, de plus la dette extérieure était quasi nulle cela était dû à la hausse des prix des hydrocarbures (Frédéric Teulon \& Dominique Bonet Fernandez, 2014), en terme d'infrastructure on remarque une modernisation des infrastructures de base et des activités de transport en Algérie. Etant donné que pour améliorer le développement humain qui est un élément essentiel du développement durable il faut améliorer les infrastructures sociales. Notre pays compte $107000 \mathrm{Km}$ de route (72\%sont revêtues) $4949 \mathrm{Km}$ de voies ferrés, 10 ports commerciaux le long de la cote ,11 aéroport internationaux et 22 aéroports nationaux (Achour Yamina, 2014).

L'Algérie espère par un double plan investissement budgétaire et assainissement de l'environnement économique à réduire la vulnérabilité structurelle et améliorer l'attractivité pour les investissements privés (Y.Ben Abdellah, 2008), mais Malgré que l'Algérie jouit d'un parc appréciable, mais des goulets d'étranglement d'ordre institutionnel et réglementaire présent sur les ports, les routes et le transport urbain et freinent la croissance. La rentabilité économique des infrastructures ferroviaires et aéroportuaires, guidée par des impératifs sociaux, reste discutable en raison de la faiblesse $\mathrm{du}$ trafic, et la compétitivité du secteur portuaire reste insuffisante par manque de terminaux à conteneur (BAFD/OCDE, 2008).

Et quand on parle de développement humain, d'après le dernier rapport du Programme des Nations Unies pour le développement PNUD sur le développement humain, l'Algérie est classé parmi les 10 nations ayant réalisé les plus grandes avancées en matière d'indice de développement humain depuis 1970,on note 75 ans pour l'espérance de vie à la naissance, $73 \%$ est le taux total d'alphabétisation des adultes, RNB par habitantes de 8,370 USD on note aussi que l'indice de développement humain IDH est de 0,713 ce qui permet à l'Algérie de se classé 93éme sur 186 pays (PNUD, 2014).

\section{Etude Empirique}

Cette étude a été inspire de celle effectué en 2004 par Gebremeskel H. Gebremariam Tesfa G. Gebremedhin, et Randall W. Jackson, intitulé: «the role of small business in economic growth and poverty allevation in west verginia: an empirical analysis». 
L'objectif principal de l'étude était d'évaluer empiriquement les rôles critiques

des petites et moyennes entreprises (PME/PMI) dans la croissance économique et la réduction de la pauvreté en Virginie-Occidentale.

La méthodologie employée implique l'analyse macroéconomique des liens entre le développement de la petite et moyenne entreprise, la croissance économique et l'incidence de la pauvreté en Virginie-Occidentale. L'objectif était d'étudier l'impact des petites et moyennes entreprises sur le taux de croissance économique et sur l'incidence de la pauvreté, tout en contrôlant simultanément pour d'autres facteurs macro-économiques affectant la croissance économique et le niveau de pauvreté.

Pour notre part notre part, nous avons pris l'équation de l'estimation de la pauvreté, étant donné que l'objectif de notre étude est de faire une estimation du niveau de vie en Algérie depuis les reformes portantes sur la promotion des PME/PMI en 2001, pour cela nous sommes référé au modèle cité ci-dessus.

Pour l'estimation du niveau de bien être, nous avons utilisé l'IDH «Indice de Développement Humain », qui est une notion apparue en 1990 sous l'impulsion des économistes Mahbub ul Haq et Amartya Sen. Cet indice est un indice composite qui s'intéresse à la satisfaction des besoins fondamentaux des individus (Gadrey \& Jany-Catrice, 2005).

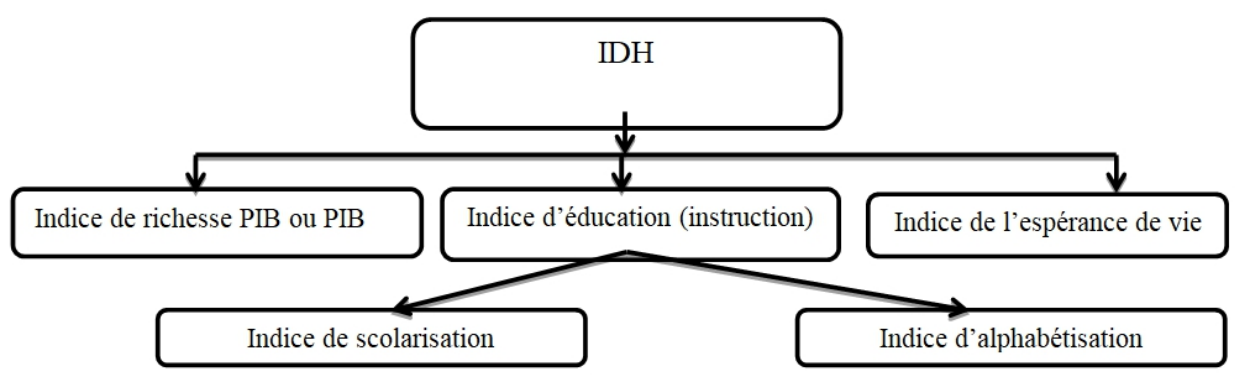

\section{Explication et Sources de Données}

Etant donné que les réformes sur les PME/PMI avaient pour principal but la création et la promotion des PME/PMI, donc les aides publiques au développement destinés à cela contribuaient à l'accroissement du nombre d'emplois dans les PME/PMI et réduisaient le chômage, et ce processus engendrait l'amélioration de l'IDH.

Notre modèle est présenté comme suit:

$\mathrm{IDH}=\alpha+\beta_{1} \mathrm{CROI}+\beta_{2} \mathrm{APD}+\beta_{3} \mathrm{PME} / \mathrm{PMI}+\beta_{4} \mathrm{CHOM}+\beta_{5} \mathrm{EMP}$ Avec:

IDH : Indice de développement humain. 
- $\quad$ CROI : CROISHH : croissance hors hydrocarbures, on l'a calculé à partir de la part des différents secteurs hors hydrocarbures du PIB (agriculture, BTPH, industrie hors hydrocarbure, transport, communication, services, et commerce).

CROISHH $=\frac{P I B h h_{t}-P I B h h_{t-1}}{P I B h h_{t-1}}$, avec PIBhh : PIB hors hydrocarbures

APD : Aides publiques au développement.

PME/PMI : Nombre des PME/PMI créent par les dispositifs mis en place par l'état pour la création des entreprises.

CHOM : Taux de chômage.

EMP : Emplois dans les PME/PMI.

- Les données ont été essentiellement récolté à partir de la banque mondiale, des publications du ministère de l'industrie et de la petite et moyenne entreprise, et des publications de L'ONS «Office national des statistiques », et du rapport du PNUD.

\section{Stationnarité des Variables}

Les résultats de l'analyse de la stationnarité relative aux variables du modèle sont résumés dans le tableau suivant:

Tableau $\mathrm{N}^{\circ}$ 3. Teste de stationnarité

\begin{tabular}{|c|c|c|}
\hline Variable & Niveau & Signification \\
\hline Idh & $3^{\text {rd }}$ & 0.00 \\
\hline Crois & $3^{\text {rd }}$ & 0.0018 \\
\hline Apd & $3^{\text {rd }}$ & 0.0003 \\
\hline PME/PMI & $3^{\text {rd }}$ & 0.0052 \\
\hline Chom & $3^{\text {rd }}$ & 0.0021 \\
\hline Emp & $3^{\text {rd }}$ & 0.0003 \\
\hline
\end{tabular}

Source : effectué à partir des résultats du traitement de données par eviews

D'après le teste de stationnarité les variables sont toutes stationnaires au troisième niveau comme nous le montre la probabilité obtenue dans le tableau au-dessus.

\section{Remarque}

Nous avons introduit le logarithme pour rendre les variables linéaire, comme dans le modèle de référence.

\section{Estimation de L'équation}

En utilisons le modèle de régression multiple, avec le logiciel eviews on obtient l'équation suivante: 


\section{Lidh= 3.84 - 0.16 lcroi + 0.13 lapd + 0.036 IPME/PMI - 0.48 lchom - 0.22 lemp}

\section{Interprétation économique et statistique}

- $\quad \mathrm{R}^{2}$ est égale à 0.903 , il se rapproche de 1 , ainsi que F-statistique il est égale à 0.001 , il est inférieur à 5\% ce qui signifie que le modèle est robuste statistiquement.

- $\quad \mathrm{R}^{2}$ ajusté est égale à 0.83 cela veut dire que $83 \%$ des variables explique la croissance hors hydrocarbures et $17 \%$ est expliqué par d'autre facteurs.

- Une hausse de la croissance économique hors hydrocarbures de $1 \%$ conduit à une baisse de l'IDH de 0.16 , c'est vrai que cela parait illogique mais il peut être expliqué par le fait que la croissance engendré par les secteurs hors hydrocarbure n'est pas bien gérer c'est-à-dire que le revenu n'est pas partager équitablement et l'un des principaux objectif du développement est l'égalité (Philippe Deubel, 2008).

- Une augmentation des aides publiques aux développement de $1 \%$ conduit à l'augmentation de l'IDH de 0.13 cela est expliqué par le fait que ces aides publiques sont en majorité destinées à la création, la mise à niveau et la promotion des PME/PMI, donc ces dépenses faites par l'état ont un rendement aussi ces aides publiques sont destinés à l'amélioration de du secteur de la santé, et le secteur de l'éducation, tout cela se manifeste par l'effet positif sur le niveau du bien-être .

- Une augmentation des entreprises crées de $1 \%$ conduit à une amélioration de l'IDH de 0.036 , comme nous nous l'avons remarqué au cours des dernières années ils ont créé des salles de soin et des dispensaires dans les zones rurales et dans les grandes villes nous avons remarqué la création des cliniques privée, tout comme nous avons remarqué l'apparition des écoles privées et cela contribue non seulement à l'amélioration des secteur de la santé et l'éducation et engendre de nouveaux emploi ce qui conduit à l'augmentation du PIB par tête, donc la création d'entreprise à un impact positive sur l'IDH.

- la réduction du chômage de $1 \%$ conduit à augmentation de l'IDH de 0.48, étant donné que l'insertion des chômeurs à la vie active engendre une augmentation du revenu national, et donc elle améliore le niveau de vie et réduit la pauvreté.

- $\quad$ l'augmentation de l'emploi par 1\% conduit à une baisse de 1'IDH de 0.22 ,cela parait illogique sauf que c'est le cas en Algérie, cela est expliquer par le fait que l'Algérie a mis en œuvre des dispositifs pour la lutte contre le chômage comme par exemple les contrats de l'ANEM et la DASS, les jeunes sont déclarés comme employés dans les entreprises sans qu'ils aient à 
travailler étant donné que c'est l'état qui paye leurs salaires et donc ils ne contribuent pas à la productivité et comme c'est des charges de plus pour l'état sans rendement c'est ainsi que s'explique le fait que le coefficient de l'emploi soit négatif, autrement dit il est vrai que la création d'emploi aide assure un revenue sauf que si cette main d'œuvre avait une valeur ajouté sur les secteur de l'éducation et de la santé elle aurait eu un impact positif sur l'IDH mais dans ce cas ces emploi créés sont des ressources inactives.

- Testes statistiques et interprétation

- Le test de Durbin Watson

Pour mieux interpréter et analyser cette statistique, nous consultons la table de DW.

On teste l'hypothèse :

$\mathrm{H}_{0}$ : il n'y a pas d'auto corrélation positive des résidus

$\mathrm{H}_{1}$ : il y a une auto corrélation positive des résidus (une erreur positive succède à une erreur positive)

Pour un nombre de variables égale à 6, la taille de l'échantillon est égale à 13 et le seuil de signification de $5 \%$ on a obtenu une valeur de DW tabulé:

$\mathrm{d} 1=0.45$

$\mathrm{d} 2=2.47$

La statistique DW est égale à 2.40, elle supérieure e à 0.45 , donc on accepte 1'hypothèse nulle $\mathrm{H}_{0}$.

Donc, Sur le plan statistique tous les paramètres sont significatifs ainsi que le coefficient de détermination et la statistique de Durbin Watson qui montre qu'il n y a pas d'auto corrélation des erreurs.

\section{Test d'heterocédasticité}

Ce teste nous permet de vérifier si les résidus (termes d'erreur) du modèle ont la même variance, c'est-à dire on vérifie si les erreurs conservent une variance constante tout au long de la période.

Tableau $\mathrm{N}^{\circ} 4$. Résultats du Test d'heterocédasticité

Heteroskedasticity Test: Breusch-Pagan-Godfrey

\begin{tabular}{cccc}
\hline \hline & & & \\
F-statistic & 1.505871 & Prob. F(5,7) & 0.2999 \\
Obs*R-squared & 6.736817 & Prob. Chi-Square(5) & 0.2410 \\
Scaled explained SS & 2.158473 & Prob. Chi-Square(5) & 0.8268 \\
& Source : résultat obtenue par eviews. &
\end{tabular}

La probabilité du test 0.2410 , elle est supérieure à $5 \%$, ce qui veut dire qu'on accepte l'hypothèse nulle d'homoscédasticité des résidus ou termes d'erreurs. 
La spécification du modèle à correction d'erreur est donc valider.

\section{Test de Normalité}

Le test de Jaque et Berra permet de vérifier la normalité d'une distribution statistique.

\section{$\underline{\text { Résultat du teste de normalité }}$}

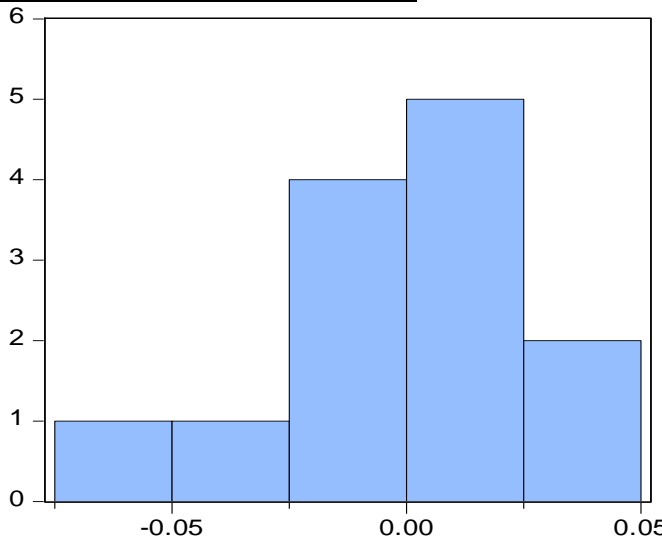

Source : résultat obtenu à partir d'eviews
Series: Residuals

Sample 20012013

Observations 13

Mean

$-6.73 e-17$

Median $\quad 0.000239$

Maximum $\quad 0.047189$

Minimum $\quad-0.061116$

Std. Dev. $\quad 0.028410$

Skewness $\quad-0.226012$

Kurtosis $\quad 3.210103$

Jarque-Bera $\quad 0.134587$

Probability

Dans le tableau de droite, nous avons les trois statistiques:

Skewness, Kurtosis et Jarque- Bera

- $\quad$ Le Jarque- Bera a une probabilité de 0,93 qui est supérieure à $5 \%$, ce qui Veut dire qu'on accepte l'hypothèse nulle de normalité des termes d'erreurs ou résidus.

- $\quad$ Le skewness est une mesure de l'asymétrie de la distribution de la série autour de sa moyenne. Pour une loi normale, le coefficient de skewness - ou coefficient d'asymétrie- est égale à zéro et le moment est d'ordre 3. Le skewness négative (-0.22) signifie que la distribution à une queue allongée vers la gauche.

- Le kurtosis ou le coefficient d'aplatissement mesure le caractère pointu ou plat de la distribution de la série. Le coefficient de kurtosis ou coefficient d'aplatissement vaut 3. La statistique de kurtosis égale à 3.21 est supérieure à 3 , la Répartition de la courbe est oscillatoire.

\section{Conclusion}

En 2001, l'état Algérien a adopté une nouvelle stratégie pour la réduction de la pauvreté et assuré un développement durable, cela en créant des dispositifs d'aide à la création des PME/PMI, et cela a porté effectivement ses fruits étant donné que la création des PME/PMI à travers plusieurs dispositifs mise en place a conduit à la réduction du chômage et à améliorer le niveau de bien-être. 
Après avoir effectué l'étude économétrique en utilisant la régression multiple pour voir l'impact des stratégies adoptées par l'Algérie sur le niveau du développement humain, nous sommes arrivés à déduire qu'il existe une relation faible mais positive entre la création des PME /PMI et l'amélioration de l'indice de développement humain, étant donné qu'elles sont le déclencheur du processus de l'amélioration du niveau du bien être en offrant une solidité a l'économie en général et le pouvoir d'achat des ménages en particulier. Malgré les efforts de l'état pour encourager la création et la mise à niveau des PME/PMI, cela reste insuffisant a cause de beaucoup de contraintes soit a priori comme le montage administratif, l'assiette foncière, la fiabilité de l'étude technico-économique ou à posteriori comme l'accompagnement de ces PME/PMI. à partir de cela, nous pouvons suggéré quelques recommandations qui se résument à contrôler les activités des PME/PMI, diversifier les activités, encourager l'internalisation de ces PME/PMI , organiser des séminaires et des formations en faveurs des jeunes entrepreneurs, en matière de gestion et enfin encourager l'innovation pour pouvoir réveillé l'esprit entrepreneurial.

\section{References:}

1. Achour Yamina (2014). Analyse de la croissance économique en Algérie, thèse de doctorat, université de Tlemcen.

2. Ahmed Smahi, Samir Maliki, \& Salah Eddine Arif (2011). La microfinance et la pauvreté subjective en Algérie: essai d'analyse, Researchgate.

3. BAFD/OCDE (2008). Perspectives économiques en Afrique.

4. Betty Wampfler (2004). La micro finance : un outil de développement durable ?, document de travail HDR, Septembre 2004, lien consulté le : 20/11/2015: http://microfinancement.cirad.fr/fr/news/Bim/Bim-2004/BIM-08-0904.pdf

5. Bulletin d'information statistique de la PME/PMI $-1^{\text {er }}$ SEMESTRE (2013).

6. Djemai Sabrina (2013). Les PME/PMI exportatrices : une croissance économique hors hydrocarbure, communication du colloque international évaluation des programmes d'investissement publique 2001-2014 et leurs retombés sur l'emploi, l'investissement, et la croissance économique, université Sétif 1.

7. Farida Merzouk (2010). PME/PMI et compétitivité en Algérie, Université de Bouira.

8. Frédéric Teulon (2014). Dominique Bonet Fernandez, Pays riche, population pauvre : quelle stratégie de développement pour l'Algérie ?, IPAG Business School. 
9. Gadrey, J. \& Jany-Catrice, F. (2005). Les nouveaux indicateurs de richesse, Editions La Découverte, Collection « Repères ».

10. Journal officiel de la république Algérienne (2001). $\mathrm{N}^{\circ} 77$ du 15 Décembre, 30Ramadhan 1422.

11. Karim Si Lekhal, Youcef Korichi, \& Ali Gaboussa (2013). Les PME/PMI en Algérie: état des lieux contraintes et perspectives, revue.univ-oeurgla.dz, $\mathrm{n}^{\circ}: 04$.

12. Khaled Sadaoui \& Mokhtar Maazouz (2009). Au-delà de la lutte contre la pauvreté, un projet de développement humain, Recherches économiques et managériales, $\mathrm{N}^{\circ} 5$.

13. Ludovica Ioana Savlovschi \& Nicoleta Raluca Robu (2011). The Role of SMEs in Modern Economy Economia. Seria Management Volume 14, Issue 1.

14. Mehdi Abbas (2012). L'ouverture commerciale de l'Algérie : Apports et limites d'une approche en termes d'économie politique du protectionnisme. Revue Tiers Monde, Presses Universitaires de France. Paris, pp.51-68. halshs-00717593.

15. Philippe Deubel (2008). Les caractéristiques du sous-développement, Analyse économique et historique des sociétés contemporaines, Pearson Education France.

16. PNUD (2000). Etude d'affinement de la carte de la pauvreté de 2000 Communes pauvres : territoires, populations et capacités d'action.

17. Rapport du PNUD sur l'Algérie (2014).

18. Romina Boarini, Åsa Johansson \& Marco Mira d'Ercole (2006). Les indicateurs alternatifs du bien-être, cahier statistique $\mathrm{N}^{\circ} 11$, OCDE.

19. Y.Ben Abdellah (2008). Le développement des infrastructures en Algérie, colloque international sur l'émergence et l'ouverture en méditerrané, Rebat. 